Classification

Physics Abstracts

$61.70 \mathrm{~J}-61.70 \mathrm{~L}-62.20 \mathrm{~F}$

\title{
In situ straining experiments in weak-beam conditions
}

\author{
D. Caillard, A. Couret, G. Molenat and J. Crestou
}

CEMES-LOE/CNRS, 29 rue Jeanne Marvig, 31055 Toulouse Cedex, France

(Received January 26, 1993; accepted April 6, 1993)

\begin{abstract}
Résumé . - Des expériences de déformation in situ sont décrites dans des situations typiques où la résolution du faisceau faible est nécessaire. Les effets de surface apparaissent peu importants, bien que l'épaisseur observée soit plus faible qu'en conditions classiques de champ clair.
\end{abstract}

\begin{abstract}
In situ straining experiments are described in some typical situations where the resolution of weak-beam is needed. Surface effects are shown to be unimportant, although the foil thickness is less than those in the classical bright field conditions.
\end{abstract}

\section{Introduction.}

The advantage of in situ straining experiments in transmission electron microscopes lies in that it provides unique information on the dynamic behaviour of crystalline defects, especially dislocations, under the combined effect of stress and temperature. Such information is essential for a sound understanding of the mechanical properties of materials, to complete results of conventional macroscopic deformation experiments, and post-mortem observations of the dislocation microstructure after deformation. It allows us, for instance, to identify mobile and sessile dislocations, and to characterize qualitatively and quantitatively the dislocation movements [1].

The artefacts which may arise during these experiments have to be carefully analysed however before conclusions can be drawn concerning the true dislocation behaviour responsible for the mechanical properties. As discussed in $[1,4]$, most artefacts originate from the use of thin foils, owing to the elastic interaction between dislocations and the free surfaces. Several critical sample thickness can be defined, depending on the physical process studied. For instance, in pure aluminium, the thickness should be larger than $0.2 \mu \mathrm{m}$ to enable cell formation, and larger than $0.8 \mu \mathrm{m}$ to generate the same dislocation density as in the bulk material (see $[3,4])$. In practice, collective dislocation behaviour can be reproduced only in thick specimens. The conditions for studying elementary processes of dislocations are however often less restrictive. In the case of individual dislocation movements, the effect of image forces is indeed preponderant, at least for dislocations not intersecting free surfaces. A critical distance between moving dislocations and free surfaces has been defined, above which image forces can be neglected. This distance is of the order of the radius of curvature of the same dislocations under the local shear stress $\sigma[1,2]$ 
( $R=\frac{\tau}{\sigma b} \approx \frac{0.5 \mu b}{\sigma}$ where $\tau$ is the line tension of dislocations, $\mu$ the shear modulus, and $b$ the Burgers vector). Under such conditions, dislocations lying far enough from the surfaces are expected to behave as in the bulk material. It is also possible to use the same criterion for studying individual dislocation movements within a substructure typical of bulk deformation, in predeformed specimens ( $\$ 3)$.

In many situations, however, it is necessary to perform in situ observations with a resolution as high as possible, i.e. using the weak-beam technique commonly employed for post-mortem observations. Examples of in situ experiments in weak-beam conditions are given in this article, with an emphasis on the original results obtained. Observations in weak-beam conditions can however be performed only in the thinnest parts of the microsamples where the image forces can be important. It is demonstrated that the criterion for neglecting the image forces is met in all cases, and that the experimental results are in agreement with the corresponding post-mortem observations and the macroscopic data.

\section{Experimental technique.}

In situ experiments in weak-beam conditions have been performed on two electron microscopes, a Philips EM-300 operating at $100 \mathrm{kV}$ and more recently a JEOL $200 \mathrm{CX}$ operating at $200 \mathrm{kV}$. Commercial straining holders have been used at room temperature, and straining holders designed in our laboratory have been used at lower and higher temperatures. Microspecimens are also described in [1] (same issue).

Observations are always made around the thin areas near the perforation of micro-specimens, where the stress is concentrated [1]. Under such conditions, the local tensile axis is close to the tensile holder axis. It is important that the perforation is nearly circular and without microcracks, especially at places where the stress is concentrated and observations are made.

In order to maintain the required weak-beam conditions, the microscope settings have to be often adjusted, owing to the rotation of the crystal lattice during the deformation. The images are recorded using a low dose camera and a magnetoscope. Since the images are usually very dark, their contrast has often to be enhanced using an image processing device. Under such conditions, dynamic sequences can be recorded at different temperatures with a resolution of the order of $2 \mathrm{~nm}$.

\section{Creep of aluminium.}

3.1 RESULTS AND INTERPRETATIONS. - Creep of metals at intermediate temperatures has been extensively studied several years ago, in order to explain deviations with respect to creep at high temperatures. In particular, it was necessary to understand the higher stress dependence of the creep rate (the so-called "power law breakdown" phenomenon), and the activation energies different from those for self diffusion (see for instance [4-8]).

At a microscopic scale, secondary creep occurs by the movement of dislocations interacting with low angle subboundaries. In situ deformation experiments have been conducted on precrept aluminium polycristals in order to study this interaction process. The first experiments were performed in a high voltage electron microscope in order to observe thick samples and to minimize the surface effects [9bc]. However, owing to the small mesh size of subboundaries $(\sim 10 \mathrm{~nm})$, it was not possible to observe the interaction mechanisms in details. In situ experiments in weakbeam conditions were thus conducted in a Philips EM-300 electron microscope. Series of very long relaxations have been performed, corresponding to very low strain-rates $\left(\dot{\varepsilon} \leq 10^{-7} \mathrm{~s}^{-1}\right)$. 
Some original pictures of dislocations interacting with subboundaries are presented below. All subboundaries have tilt and twist components and no long-range stress field, as demonstrated in [9a].

Figure 1 illustrates the extraction process of a dislocation from a subboundary at room temperature. It leads to a distorsion illustrated schematically in $c$ (open circles correspond to dots), which disappears very slowly by climb (after $t=232$ minutes). The whole process is described in figure $1 \mathrm{~b}$, which correspond to figure $1 \mathrm{c}$ when dislocations of Burgers vector $b_{1}$ are out of contrast. Another example of this process can also be found in [10]. It is worth noting that this extraction process is in good agreement with the results obtained previously in HVEM [9c].

Figure 2 illustrates the insertion process of gliding dislocations in the same subboundary. The inserted dislocation is underlined in figure $2 \mathrm{~b}$ by dots which correspond to open circles in figure $2 \mathrm{a}$. This sequence shows that dislocations are inserted preferably at vacant places where the mesh is larger, often resulting from previous extraction processes. Additional examples of the same behaviour can be found in [10]. It can be readily explained by the attractive local stress field bound to the larger mesh. Insertion processes often give rise to distorsions in the periodicity of the dislocation network [10].

A strong correlation has been found between insertion and extraction processes, by plotting the positions of all inserted and extracted dislocations on the same drawing (Fig. 3, from [10]). Extraction processes are seen to occur preferably close to the positions of the previously inserted dislocations, in the same way as insertion processes are seen to occur at vacant places set free by previous extraction processes. This memory effect is a consequence of the slowness of the climb process which tends to smooth out all the distortions following the extraction (Fig. 1a) and insertion processes (Fig. 2b). It is even possible to determine the exact extraction sites, since they can be identified with the coincidence sites (arrowed) between inserted and extracted dislocations.

All these results have been used to formulate a model of creep at intermediate temperatures [11].

3.2 DisCUSSIONS OF THE POSSIBLE ARTEFACTS. - The experiments presented in $\S 3.1$. have been carried out at $100 \mathrm{kV}$, and as a result the effect of irradiation was unimportant. The foil thickness was however of the order of $300 \mathrm{~nm}$, i.e. much smaller than for bright field HVEM observations $(2 \mu \mathrm{m})$. It is thus necessary to discuss the effect of image forces.

The compatibility between results of weak-beam observations at $100 \mathrm{kV}$ and bright field observations at $1 \mathrm{MeV}$ is a first indication that the surface effects may not be very large. It is however necessary to check whether the criterium explained in $\S 1$ is met or not. The mechanism controlling creep is the extraction process from subboundaries. The local stress necessary to initiate extraction has been estimated to be much larger than the applied creep stress [12]. It is of the order of the Orowan stress corresponding to the subboundary mesh size, i.e. $\sigma \approx 0.5 \frac{\mu b}{h}$, where $\mu$ is the shear modulus, $b$ the Burgers vector, and $h$ the subboundary mesh size [12]. The corresponding radius of curvature is $R \approx h$, as directly measured in [12] ( $R=30 \mathrm{~nm}$ in this case). Since the distance between the extraction sites and the surfaces is of the order of $100 \mathrm{~nm}$, i.e. larger than $h$ and $R$, the effect of image forces is seen to be negligible.

\section{Glide mechanisms in nickel-based intermetallic alloys.}

4.1 RESUlTS AND INTERPRETATIONS. - Intermetallic alloys have many potential applications owing to their high mechanical strength at high temperature. The plastic properties of these materials are different from those of pure metals mainly because dislocations are paired, i.e. the 

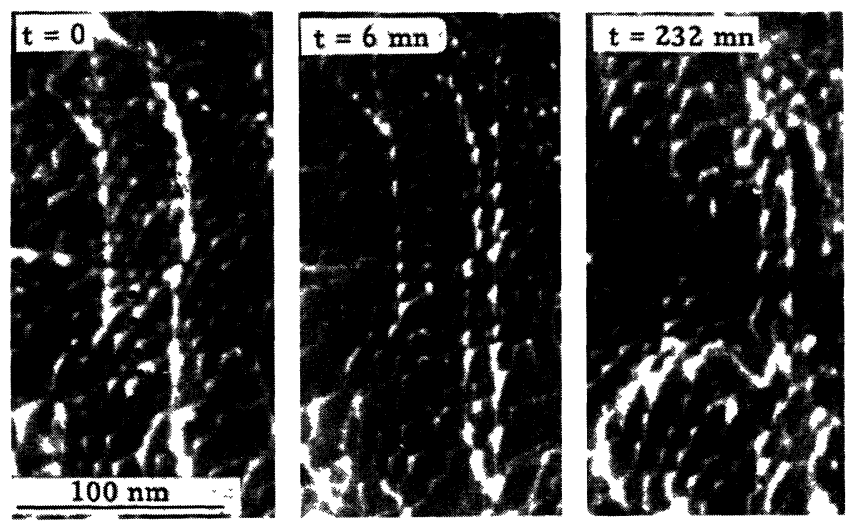

a)
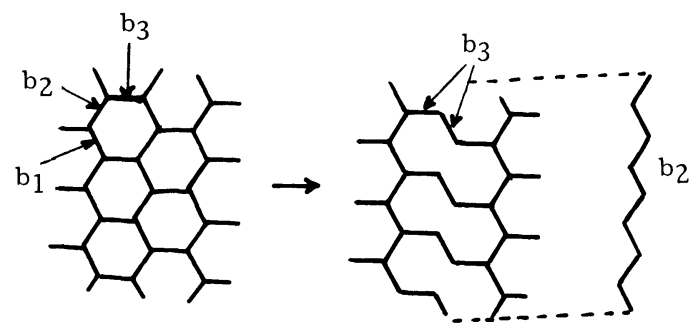

b)
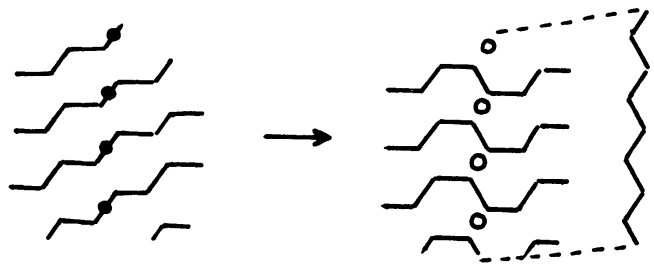

c)
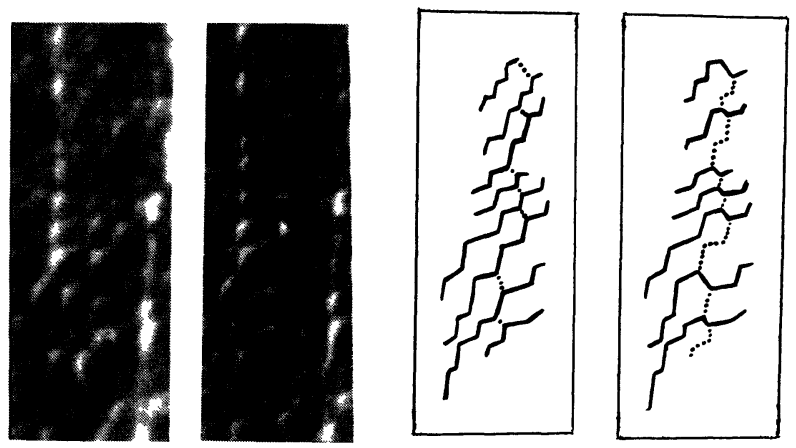

d)

Fig. 1. - Creep of aluminium: extraction process from a low angle subboundary at room temperature $100 \mathrm{kV}$. a) dynamic sequence in weak-beam condition. b) schematic diagram. c) same as $b$, with dislocations $b_{1}$ out of contrast. d) enlargement of the central parts of two pictures $(t=0$ and $t=6 \mathrm{mn})$, and schematic diagram. The extracted dislocation is in dotted lines. 

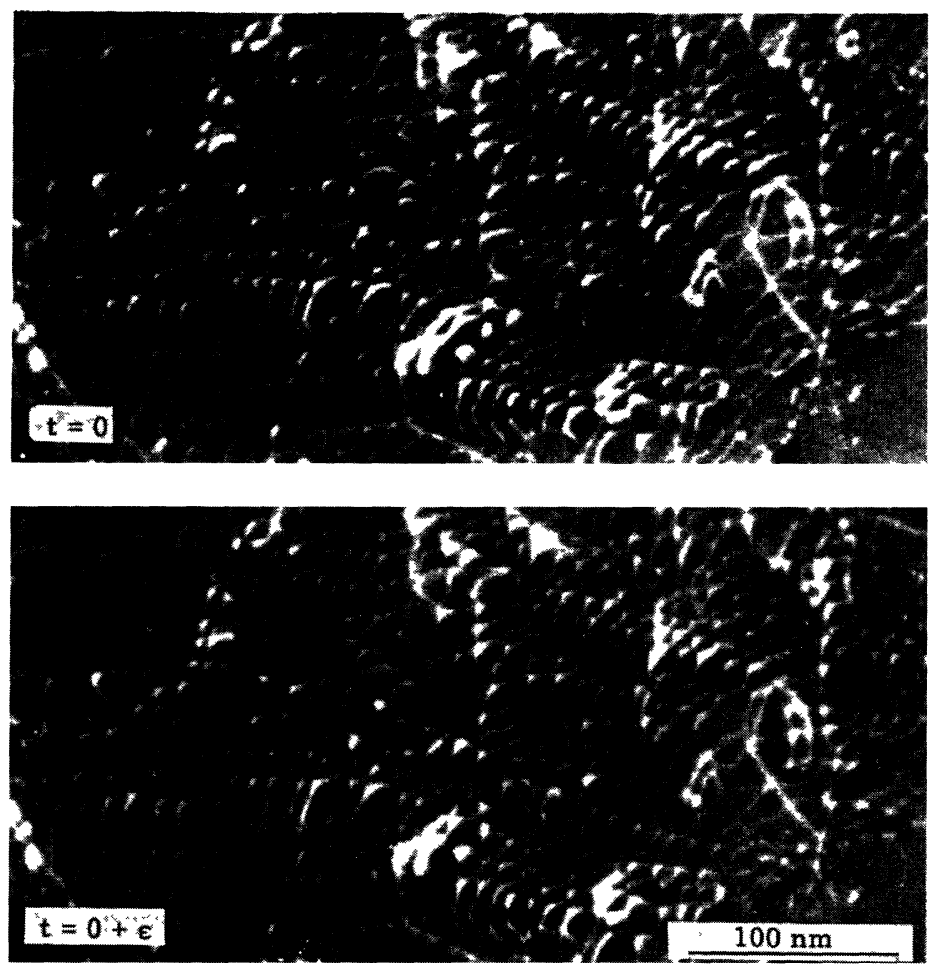

Fig. 2. - Creep of aluminium: insertion process inside a low angle subboundary at room temperature $100 \mathrm{kV}$, weak-beam condition. The inserted dislocation is underlined by dots in $\mathrm{b}$ corresponding to open circles in a.

so-called superdislocations are made of two superpartial dislocations similar to those of the disordered structure, separated by a ribbon of antiphase boundary (APB). Since the APB ribbon does not necessarily lie in the glide plane, rather complex mechanisms occur which control the plastic properties of intermetallics. It is thus important to image the two components of superdislocations during deformation. In $\mathrm{Ni}_{3} \mathrm{Al}$ and nickel-based intermetallic alloys, the dissociation width of superdislocations ranges between 4 and $6 \mathrm{~nm}$, as a result the weak-beam technique is absolutely necessary. Original results are presented in the case of glide in $\{111\}$ (octahedral) planes.

Figure 4 exhibits a rectilinear screw superdislocation in a $\mathrm{Ni}_{3} \mathrm{Al}$ single crystal strained at room temperature. It is dissociated into two superpartial dislocations and glides in a $\{111\}$ plane in agreement with results given by other techniques. Tilting experiments show that the APB ribbon lies in the $\{111\}$ glide plane (see also [13]). This dislocation experiences a high frictional force since it moves at a much lower velocity than the edge segments in the same plane. Its movement is however not steady as in the case of the Peierls mechanism, but jerky. Moreover, it proceeds by jumps over distances which are exactly scaling with the width of the APB ribbon, as a result the trailing superpartial dislocation takes the exact place of the leading. Macrokinks (MK) are sometimes observed after one jump. Other examples of the same process have been presented elsewhere $[14,15]$. These results are new since screw superdislocations were expected to be very unstable in the $\{111\}$ glide plane and to cross slip very easily in the $\{100\}$ plane. Moreover screw dislocations dissociated in the $\{111\}$ glide plane were not expected to experience a frictional force. These results have been modelled and included in a new model of yield stress anomaly [15]. 


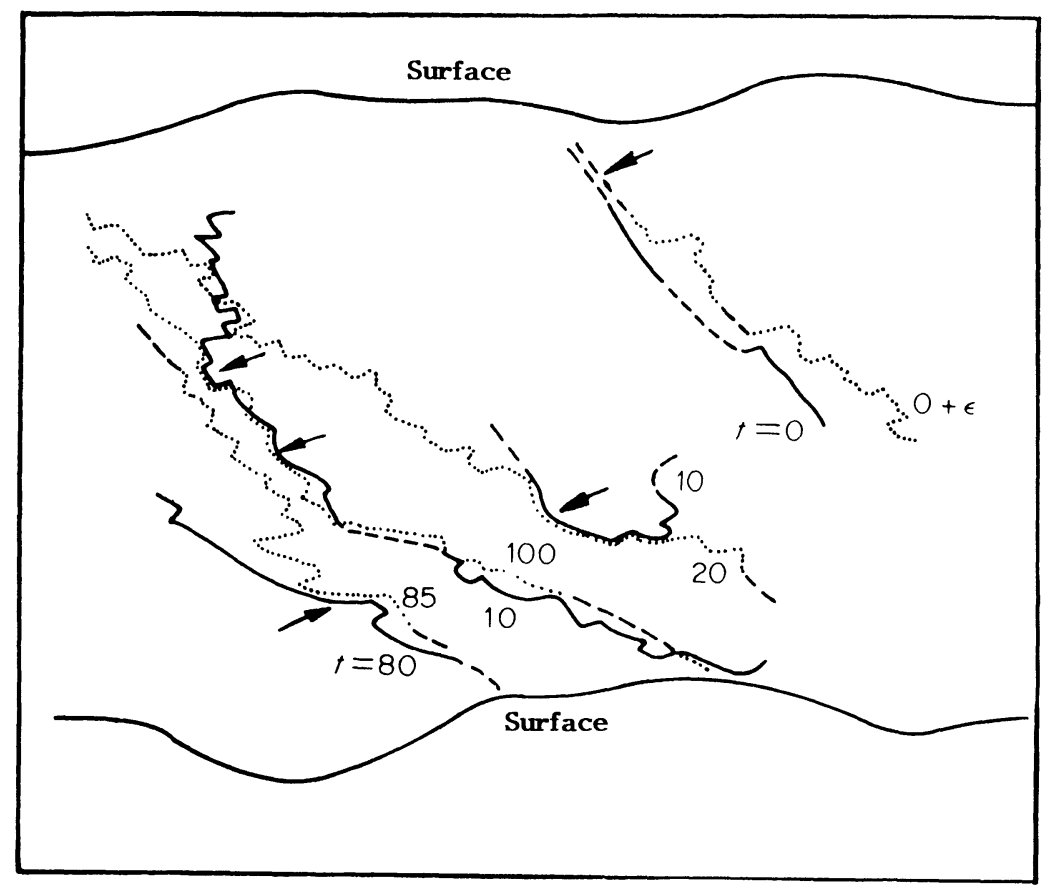

Fig. 3. - Creep of aluminium: memory effect (from [10]). Solid lines: positions of inserted dislocations. Dots: positions of extracted dislocations. Numbers refer to the time ( $\mathrm{mn}$ ) of the corresponding events. Arrows point to extraction sites.

Additional in situ weak-beam observations have been made in intermetallics in the case of glide in $\{100\}$ planes of $\mathrm{Ni}_{3} \mathrm{Al}$ at $873 \mathrm{~K}$ [16], glide in $\{100\}$ planes of the $\gamma^{\prime}$ phase of a superalloy at $740 \mathrm{~K}$ [2], and glide in prismatic planes of $\mathrm{Ti}_{3} \mathrm{Al}$ at $300 \mathrm{~K}$ [17]. They have yielded information on the frictional forces, and the interaction mechanisms with clusters or solute atoms.

4.2 Discussion of THE POSSIBLE ARTEFACTS. - In the case of glide in the $\{111\}$ planes of $\mathrm{Ni}_{3} \mathrm{Al}$, the main conclusions of in situ experiments have been confirmed by subsequent postmortem observations. Rectilinear screw superdislocations dissociated in $\{111\}$ [13] have been observed post-mortem $[18,19]$, and jumps over distances scaling with the dissociation width have also been evidenced by the post-mortem observation of a high density of macrokinks similar to MK in figure 4 [20].

In the case of the glide mechanisms in the $\{100\}$ planes of $\mathrm{Ni}_{3} \mathrm{Al}$ and $\gamma^{\prime}$ crystals, the substructure observed is very similar to that observed post-mortem, and the temperature dependence of the yield stress (estimated from dislocation radii of curvature) is also comparable to that of bulk samples (see a discussion in $[2,16,21]$ ). In addition, the thickness criterion has been shown to be met in all the cases studied.

\section{Conclusions.}

This article does not describe all the results that have been obtained in situ under weak-beam conditions. On the contrary, it is aimed to show through a few examples that heating, straining 

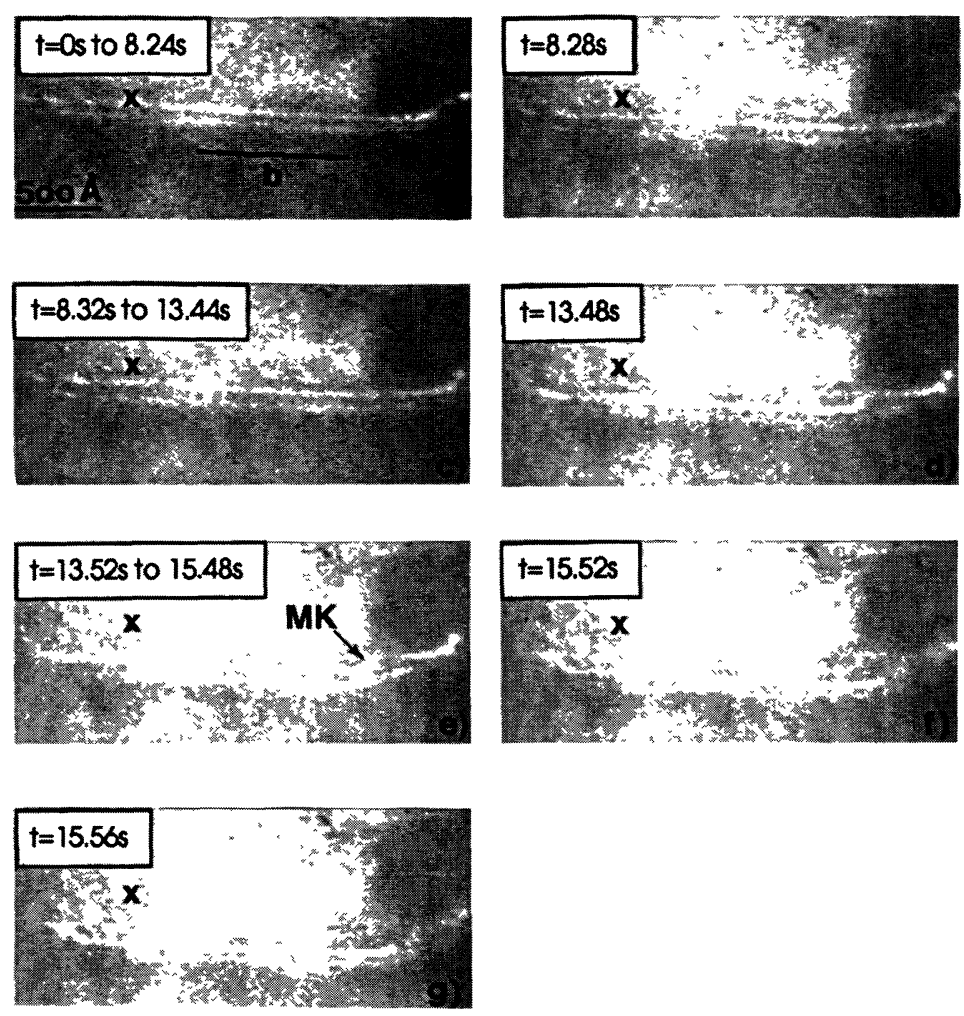

Fig. 4. - Glide in a $\{111\}$ plane of $\mathrm{Ni}_{3} \mathrm{Al}-200 \mathrm{kV}$, weak-beam condition. b: Burgers vector. MK: macrokink. $\mathrm{X}$ is a fixed point. The left hand side exhibits all the successive positions of the superpartial dislocations. The right hand side exhibits the frames corresponding to the jump events.

and observing under weak-beam conditions at the same time is quite feasible and useful to bring original informations, provided very good microsamples are used.

The comparison between the results obtained in situ, the results obtained post-mortem, and the results of conventional macroscopic deformation experiments, indicates that no strong artefact modifies the elementary processes of dislocations inside the microscope. In addition, the thickness criterion for neglecting image forces is rather easily fulfilled in many situations, even in thin foils designed for weak-beam observations at 100 or $200 \mathrm{kV}$. In practice, all light materials with a high mechanical strength (materials for industrial applications, for instance) can be strained in situ under weak-beam conditions, provided some elementary precautions are taken.

\section{References}

[1] Couret A., Crestou J., Farenc S., Molénat G., Clément N., Coujou A. and Caillard D., this issue.

[2] Clément N., Couret A. and Caillard D., Philos. Mag. A 64 (1991) 669.

[3] ImURA T., in "High Voltage Electron Microscopy", Eds. P.R. Swan, C.J. Humphreys and M.J. Goringe (Academic Press, London and New York, 1974) p.179. 
[4] MARTIN J.L. and KuBin L.P., Ultramicroscopy 3 (1978) 215.

[5] TAKeuCHi S. and ARgON A.S., J. Mat. Sci. 11 (1976) 1542.

[6] PoIRIER J.P., Acta Met. 26 (1978) 629.

[7] Siethoff H., Schroter W. and AHLbORn K., Acta Met. 33 (1985) 443.

[8] Blum W., Straub S. and Vogler S, in "Strength of Metals and Alloys", Proc. ICSMA 9, Eds. D.G. Brandon, R. Chaim and A. Rosen (Freund Publishing House Ld, London) 1 (1991) p. 111.

[9] Caillard D. and Martin J.L., a) Acta Met. 30 (1982) 437, b) Acta Met. 30 (1982) 791, c) Acta Met. 31 (1983) 813.

[10] Caillard D., Acta Met. 32 (1984) 1483.

[11] Caillard D., Philos. Mag. A 51 (1985) 157.

[12] Caillard D., Mat. Sci. Eng. 81 (1986) 349.

[13] Caillard D., Clément N., Couret A., Lours P. and Coujou A., Philos. Mag. Lett. 58 (1988) 263.

[14] Molénat G. and Caillard D., Philos. Mag. A 64 (1991) 1291.

[15] Molénat G., Caillard D., Couret A. and Paidar V., High Temperature Ordered Intermetallic Alloys V, I. Baker, R. Darolia, J.D. Whittenberger, M.H. Yoo Eds., MRS Symposium Proceedings 288 (1993) p. 287.

[16] Molénat G. and Caillard D., Philos. Mag. A 65 (1992) 1327.

[17] Legros M., Couret A. and Caillard D., High Temperature Ordered Intermetallic Alloys V, I. Baker, R. Darolia, J.D. Whittenberger, M.H. Yoo Eds., MRS Symposium Proceedings 288 (1993) p. 323.

[18] BonTEMPS C., Ph.D. Thesis, University of Paris-Sud, France (1991).

[19] WEN M. and LIN D., High Temperature Ordered Intermetallic Alloys IV, Eds. L.A.J. Johnson, D.P. Pope and J.O. Stiegler, MRS Symposium Proceedings 213 (1991) p. 343.

[20] Couret A., Sun Y.Q. and Hirsch P.B., Philos. Mag. A67 (1993) 29.

[21] Clément N., Molénat G. and Caillard D., Philos. Mag. A 64 (1991) 697. 